\title{
Outcome- statt Prozessorientierung in der ärztlichen Weiterbildung
}

\section{Richard O. Binswanger}

Der Autor arbeitet als Konsiliararzt für Radiologie an den Spitälern Münsterlingen und Baden.
* Im Rahmen der 2011 erfolgreich abgeschlossenen Akkreditierung der Weiterbildungsprogramme der Fachgesellschaften beim Bund überprüfte der Autor alle Selbstbeurteilungsberichte der Fachgesellschaften. Er nimmt regelmässig an Visitationen der Weiterbildungsstätten als fachfremder Experte des SIWF teil.

\section{Korrespondenz:}

Dr. med. Richard O. Binswanger Radiologie und Nuklearmedizin FMH

Führungsschule Bodensee

Münsterlingen

Oberer Seeweg 9

CH-8597 Landschlacht

r.binswanger[at]bluewin.ch

www.fsb-spital.ch
Die Weiterbildungsprogramme der medizinischen Fachgesellschaften* definieren die $\mathrm{zu}$ erwerbenden Kenntnisse und Fertigkeiten und schliessen mit einem Fachexamen ab. Insoweit sind sie outcomeorientiert. Daneben enthalten sie zahllose und immer mehr prozessorientierte Elemente, so die Weiterbildungsdauer, Fall- und Operationszahlen, Kriterien zur Einteilung der Weiterbildungsstätten. So werden zum Beispiel die Anzahl Stunden theoretischer Weiterbildung, die zu haltenden Fachzeitschriften, das Sicherheitsmanagement überprüft. Sogar die Arbeitszeiten werden kontrolliert. Immer grössere Anforderungen betreffen die Dokumentation, das Logbuch wurde soeben eingeführt. Angetrieben wird diese Entwicklung von Experten, zum Beispiel den neuen Masters of Medical Education, Juristen und den Turf Battles der Fachgesellschaften.

\section{Die Qualitätssicherung überprüft fast nur die Prozesse}

Die Visitationen der Weiterbildungsstätten sind ein wichtiges Instrument der Qualitätssicherung für das Schweizerische Institut für Medizinische Weiter- und Fortbildung (SIWF). Es ist prozessorientiert. Abgesehen vom Urteil der Weiterzubildenden kann der Outcome der Weiterbildung nicht ermittelt werden. Dazu dient das Facharztexamen. Eine Ebene höher läuft die Akkreditierung der Weiterbildungsprogramme beim Bund. Sie ist ebenfalls fast ausschliesslich prozessorientiert. Dementsprechend feilen alle Beteiligten an schönen Papieren, in denen Prozesse dargestellt werden. Und wie steht es mit der Umsetzung?

\section{Der Aufwand und seine Konsequenzen}

Er ist gigantisch. Er absorbiert gewaltige Mengen an Zeit, die der klinischen Arbeit und dem Bedsideteaching verlorengeht. Und sehr viel Geld. Die Kosten für die Akkreditierung beliefen sich auf beinahe eine Million Franken, Mittel, die, notabene, von den Gebühren der Facharztkandidaten stammen. Viel bedeutungsvoller ist aber die schleichende Veränderung der Einstellung und Haltung aller Beteiligten: weg vom Inhalt, hin zum Prozess, weg vom Patienten und an den PC. Dies prägt eine ganze Generation von Ärztinnen und Ärzten. Sie werden reglementsabhängig, vorschriftengläubig, subaltern im Denken. Selbständige und mutige Entscheidungsfindung wird behindert statt gefördert.

\section{Ein Plädoyer für die Outcomeorientierung}

Die grösste Qualitätsverbesserung hat die Schweizerische Weiterbildung durch die Einführung der Facharztprüfungen erfahren. Das Beispiel der Radiologie ist hier überzeugend. Die Radiologen sind seither um Vieles besser geworden. Deswegen: Das Facharztexamen soll zum primären Instrument der Qualitätssicherung der Weiterbildung ausgebaut werden. Mit einem Bruchteil des Aufwandes für die Erfüllung und Kontrolle der bisherigen Bestimmungen. Also weg mit dem Grossteil der prozessorientierten Kriterien und hin zu einer besseren Evaluation des Outcomes.

\section{Und die Konsequenzen?}

Die Facharztexamina müssten erweitert werden, vor allem durch eine genauere und ausführlichere Überprüfung der Fertigkeiten. Fachgesellschaften, Weiterbildungsstätten und Assistenzärzte wären von Formalien entlastet. Natürlich werden alle auch in Zukunft grosse Kräfte in die Weiterbildung stecken müssen. Die Entlastung für das SIWF wäre enorm. Die frei werdenden Ressourcen kann es in die Unterstützung und nicht nur die Kontrolle der Weiterbildungsstätten stecken.

\section{Und das SIWF?}

Den aktuellen Zustand der Weiterbildung und deren aktuelle Regelungen sehe ich als Erfolg. Der Präsident des SIWF fasst meine Überlegungen als Anregung auf und will sie in irgendeiner Weise bei den Überlegungen zur Zukunft berücksichtigen.

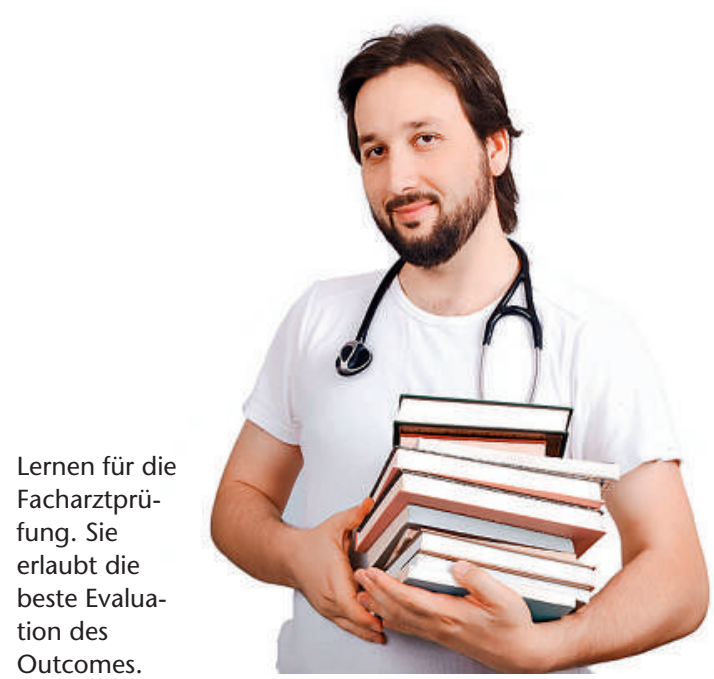

